\title{
MISSIONS AMÉRICAINES
}

Les Frontières de la mission ( $X V^{e}-X I X^{e}$ siècle), Mélanges de l'École française de Rome. Italie et Méditerranée, t. CIX, 2, Rome, École française de Rome, 1997. $17 \times 24$, p. $485-782$.

Giovanni Pizzorusso, Roma nei Caraibi. L'organizzazzione delle missioni cattoliche nelle Antille e in Guyana (1635-1675). Rome, École française de Rome, 1995. $17 \times 24,366$ p., bibliogr., index (Collection de l'École française de Rome, 207).

Jesuit encounters of the New World. Jesuit chroniclers, geographers, educators and missionaries in the Americas, 1549-1767. Sous la dir. de Joseph A. GaGliano et Charles E. Ronan, S.J. Rome, Institutum Historicum Societatis Iesu, 1997. $16 \times 24,335$ p. (Bibliotheca Instituti Historici S. I., vol. L).

Dauril Alden, The Making of an enterprise. The Society of Jesus in Portugal, its empire, and beyond, 1540-1750. Stanford, CA, Stanford University Press, 1996. 18,5 × 26, xxxviII-707 p., index, cartes, ill., tabl.

La Mission jésuite du Brésil. Lettres et autres documents (1549-1570). Éd. et trad. de Jean-Claude Laborie, en collab. avec Anne Lima. Paris, Chandeigne/Librairie portugaise, 1998. $16 \times 22,351$ p., index, bibliogr. (Magellane).

Les actes du colloque Les Frontières de la mission $\left(X V^{e}-X I X^{e}\right.$ siècle), qui s'est tenu à Rome en décembre 1992, marquent une étape importante dans la définition des objets et des problèmes de l'histoire des missions et des institutions religieuses chargées de leur promotion. Le choix d'un ample éventail chronologique permet de rendre compte de l'évolution des frontières de la mission, alors que la volonté avouée de placer sur un même plan missions intérieures et missions extérieures met en lumière la « ressemblance des situations» ainsi que leur continuité institutionnelle, notamment par l'exploration du rôle de la congrégation De Propaganda Fide. L'étude de la mission de l'extérieur comme prolongement de la mission intérieure (Dominique Deslandres) ou comme miroir des luttes confessionnelles européennes (Giovanni Pizzorusso) fonde l'idée d'une perméabilité entre les deux terrains dont il importe de comprendre les fondements spirituels ainsi que les différentes stratégies institutionnelles (Bernard Dompnier). Le lien entre ces deux aspects s'affirme pleinement dans l'étude des Litterae Indipetae de Pizzorusso: expression des conceptions spirituelles des futurs missionnaires héritées de l'idéal

Revue de synthèse : $4^{\text {e }}$ S. ${ }^{\text {os }} 2-3$, avr.-sept. 1999, p. 478-481. 
de croisade, ces documents sont au cœur de la machine bureaucratique qui régit l'organisation des missions jésuites.

Malgré la communauté d'objectifs, les méthodes et les lectures des cultures locales varient selon les ordres religieux (David Gentilcore). Ces adaptations ne vont pas dans le sens d'une acceptation de ces cultures, comme le montrent Willi Henkel pour le Pérou et Paule Brasseur pour l'Afrique de l'Ouest, mais contribuent d'une part à construire indirectement des identités particulières, celle des populations maritimes par exemple (Alain Cabantous), et d'autre part sanctionnent le processus d'accommodation comme une acculturation des missionnaires eux-mêmes (Matteo Sanfilippo).

L'évolution des missions européennes en Europe au Xvin' siècle confirme l'idée d'une croissante intégration socioculturelle et d'une pénétration en profondeur des principes de Trente mais aussi des Lumières (Louis Châtellier), alors que ce « christianisme de raison ", à travers ses institutions centrales, s'interroge sur la validité de l'esclavage hors des frontières européennes (Stefania Nanni).

Le poids des institutions ecclésiastiques sur le processus missionnaire se vérifie à des échelles diverses. À celle du diocèse, l'étude des visites pastorales et de leur interférence avec les missions permet de prendre la mesure des différentes conceptions du gouvernement ecclésiastique, selon les orientations de recherche proposées par Adriano Prosperi. À l'échelle du Saint-Siège, les archives de la congrégation $D e$ Propaganda Fide fournissent, d'une part, un point de vue complémentaire sur l'histoire d'une région comme le Liban (Elias Kattar) et contribuent à éclairer le rôle concret de Rome, et aussi son échec, dans la lutte contre le protestantisme français au XviI ${ }^{\mathrm{e}}$ siècle (Frédéric Meyer). D'autre part, cette documentation ouvre les études missionnaires sur des problématiques institutionnelles, à travers l'étude de la centralisation des informations et des processus de prise de décision. Alors que Bernard Heyberger montre que pour le Levant au Xvirl siècle "l'expérience de la périphérie ne modifie que très peu la vision à partir du centre romain ", Claude Prudhomme insiste sur la croissante centralisation romaine et l'imposition aux missionnaires d'un modèle romain, qui révèle les limites de l'adaptation dans les contextes de colonisation et des indépendances des $\mathrm{xIX}^{\mathrm{e}}$ et $\mathrm{xx}^{\mathrm{e}}$ siècles.

Comme le souligne Luca Codignola dans son article conclusif au colloque, l'histoire des processus missionnaires ne peut se résumer au constat de l'incompréhension par ses acteurs des cultures locales. Il importe de s'interroger sur «l'efficacité du travail missionnaire sur le terrain », sur « le niveau d'acculturation réciproque " entre missionnaires et missionnés et, enfin, sur « le niveau de centralisation » du travail missionnaire (p. 787).

C'est, entre autres, ce dernier aspect qui guide le travail de Pizzorusso dans Roma nei Caraibi. Visant dans un premier temps à reconstruire l'histoire des missions catholiques dans les Caraibes, l'ouvrage fait aussi le point sur l'intervention romaine dans la région. L'auteur montre comment, en quarante ans, d'une coïncidence d'intérêts entre le pape et le roi de France on passe avec Colbert à un croissant contrôle de la Couronne sur les colonies. L'étude de deux institutions religieuses centrales, la curie des dominicains à Rome et la congrégation De Propaganda Fide, démontre non seulement que les ordres religieux parviennent à garder leur indépendance vis-à-vis de la curie pontificale, mais qu'elle est tributaire de leurs informa- 
tions, alors qu'elle ne réussit pas à imposer un clergé séculier ni des structures ecclésiastiques qui lui soient directement attachés. Il en résulte que l'encadrement des fidèles dans les Caraïbes reste sous la houlette des ordres religieux jusqu'à 1850 , date de la première nomination d'un évêque.

Le recueil d'articles Jesuit encounters of the New World invite à la comparaison. Les stratégies missionnaires de la Compagnie sont abordées dans le contexte de trois puissances coloniales différentes : au Brésil (Luis Palacin Gomez), chez les Hurons (Mary Ann La Fleur) et dans le Maryland (R. Emmett Curran). Le choix de l'éducation des élites locales à Georgetown, évident aussi au Mexique (Esteban J. Palomera), est au centre de la question de l'indétermination de la vocation de la Compagnie qui se pose au Brésil (Thomas Cohen et la biographie du P. Manuel da Nóbrega par Dauril Alden). La production de chroniques (W. Michael Mathes) et de cartes (David Buisseret, Anne Godlewska) est au cœur de l'analyse de la lecture des cultures locales par les missionnaires (Nancy Bonvillain), mais aussi de l'étude des formes de résistance et d'adaptation des populations indigènes aux institutions missionnaires et coloniales (Susan Deeds), selon les orientations de la « New Mission History ", fondée sur l'étude des perspectives amérindiennes.

L'ouvrage d'Alden constitue la synthèse d'un travail de vingt ans qui vise à vérifier, documents à la main, la crédibilité du mythe de la puissance et de la richesse de la Compagnie de Jésus. Pour cela, l'auteur a choisi d'étudier l'histoire de la Compagnie dans l'assistance du Portugal, autrement dit dans l'empire portugais, depuis sa création jusqu'à la veille de son expulsion (un second ouvrage consacré exclusivement à ce thème est promis par l'auteur). Les deux premières parties et la cinquième, qui font le point sur la chronologie, retracent les relations de l'ordre avec la couronne portugaise ainsi que les étapes de son expansion territoriale dans l'Empire (Brésil, Afrique, Inde et Extrême-Orient), en mettant l'accent sur les rapports plus ou moins conflictuels avec la société portugaise, péninsulaire et coloniale, et sur l'impact sur la Compagnie des vicissitudes de l'Empire. Elles encadrent deux importants volets, centrés sur le fonctionnement de l'ordre. L'un met l'accent sur l'organisation bureaucratique et le choix de la centralisation, en insistant sur le recrutement, grâce à l'exploitation systématique des catalogues triennaux, qui fournissent de précieux tableaux statistiques. L'autre volet étudie le financement et les activités économiques de l'ordre (propriété de la terre, agriculture, commerce), considérées comme des palliatifs stables au plus qu'aléatoire financement royal. Non seulement ces activités sont au cceur de l'animosité suscitée très tôt par la Compagnie, mais elles permettent d'aborder les contradictions internes d'une institution piégée par sa prétention à l'autonomie et à l'universalité.

La Mission jésuite du Brésil est le pendant documentaire brésilien à la synthèse présentée précédemment. Itinéraire dans les vingt premières années de la présence jésuite au Brésil, ce choix de documents, édités et traduits par Jean-Claude Laborie, illustre la variété de la production épistolaire des missionnaires, depuis la collection de cas édifiants (doc. 7) jusqu'à l'exposé des tensions internes à l'ordre (doc. 13), en passant par la démonstration précise des difficultés quotidiennes (doc. 11) ou par la lettre quadrimestre, première vision globale des terres brésiliennes (doc. 12). Ce par- 
cours documentaire vise néanmoins à démontrer l'unité qui caractérise ce «seul et unique texte écrit à plusieurs mains » (p. 8), à travers des thèmes récurrents et des règles d'écriture conditionnés par l'identité jésuite des scripteurs. Toutefois, et c'est le second objectif du recueil, ce choix, circonscrit sur une vingtaine d'années, rend compte de l'évolution de la mission jésuite du Brésil vers une croissante inclusion des colons dans l'éventail des activités de la Compagnie, à mesure de son institutionnalisation. Au-delà des questions théologiques et des stratégies concrètes de conversion des Indiens, on aborde les peurs, les incertitudes et les espoirs du missionnaire lui-même, grâce au classique «Dialogue sur la conversion des gentils du père Manuel da Nóbrega » (doc. 18), inédit en français. Cette exploration du quotidien de la mission s'enrichit par touches éparses dans la correspondance d'un aperçu sans fioritures des conditions matérielles des jésuites du Brésil, de leurs activités économiques et du choix de recourir au travail des esclaves.

La volonté de restituer certains des textes dans leur version originale, antérieure à la censure pratiquée par la hiérarchie jésuite avant les publications contemporaines, est, à l'image de la présentation critique du recueil, fondée sur le souci d'aborder la mission jésuite du Brésil dans toutes ses contradictions et ses significations.

Malgré la diversité des articles et des thèmes abordés, le mérite commun de ces ouvrages est autant de mettre en lumière les rouages institutionnels de la mission que d'en approcher le fonctionnement dans toute sa complexité. L'importante place occupée par les études sur la Compagnie de Jésus, que renforcent les thèses soutenues ces derniers mois par Carlos Zerón et Charlotte de Castelnau ou en cours de réalisation de Jean-Claude Laborie et de Hervé Pennec sur le Brésil et l'Éthiopie, confirme la richesse d'un champ de recherches en renouvellement. La perspective comparatiste ouverte par les études sur la congrégation De Propaganda Fide non seulement comble un vide mais augure de prochains travaux sur les autres ordres religieux.

Aliocha MaLdavsKY (janvier 1999). 